\title{
Aromaticity of distorted benzene rings. Exploring the validity of different indicators of aromaticity
}

\author{
Ferran Feixas, Eduard Matito, Jordi Poater, and Miquel Solà* \\ SUPPORTING INFORMATION
}

Table S1. XYZ coordinates of the B3LYP/6-311++G** optimized and distorted benzene rings. $\quad$ S2

Figures S1-S6. Normalized values of the aromaticity indexes analyzed. $\quad$ S13 
Table S1. XYZ coordinates of the B3LYP/6-311++G** optimized and distorted benzene rings.

$\begin{array}{lrrr}\mathrm{C}_{6} \mathrm{H}_{6} & & & \\ 6 & 0.000000 & 1.394663 & 0.000000 \\ 6 & 1.207814 & 0.697332 & 0.000000 \\ 6 & 1.207814 & -0.697332 & 0.000000 \\ 6 & 0.000000 & -1.394663 & 0.000000 \\ 6 & -1.207814 & -0.697332 & 0.000000 \\ 6 & -1.207814 & 0.697332 & 0.000000 \\ 1 & 0.000000 & 2.479089 & 0.000000 \\ 1 & 2.146954 & 1.239544 & 0.000000 \\ 1 & 2.146954 & -1.239544 & 0.000000 \\ 1 & 0.000000 & -2.479089 & 0.000000 \\ 1 & -2.146954 & -1.239544 & 0.000000 \\ 1 & -2.146954 & 1.239544 & 0.000000\end{array}$

\section{BLA}

$\Delta \mathrm{R}=0.05$

$\begin{array}{rrrr}6 & 0.684832 & 1.215031 & 0.000000 \\ 6 & 1.394663 & -0.014434 & 0.000000 \\ 6 & 0.709832 & -1.200597 & 0.000000 \\ 6 & -0.709832 & -1.200597 & 0.000000 \\ 6 & -1.394663 & -0.014434 & 0.000000 \\ 6 & -0.684832 & 1.215031 & 0.000000 \\ 1 & 1.227044 & 2.154171 & 0.000000 \\ 1 & 2.479089 & -0.014434 & 0.000000 \\ 1 & 1.252044 & -2.139737 & 0.000000 \\ 1 & -1.252044 & -2.139737 & 0.000000 \\ 1 & -2.479089 & -0.014434 & 0.000000 \\ 1 & -1.227044 & 2.154171 & 0.000000\end{array}$

$\Delta \mathrm{R}=0.1$

$\begin{array}{rrrr}6 & 0.672332 & 1.222247 & 0.000000 \\ 6 & 1.394663 & -0.028868 & 0.000000 \\ 6 & 0.722332 & -1.193380 & 0.000000 \\ 6 & -0.722332 & -1.193380 & 0.000000 \\ 6 & -1.394663 & -0.028868 & 0.000000 \\ 6 & -0.672332 & 1.222247 & 0.000000 \\ 1 & 1.214544 & 2.161387 & 0.000000 \\ 1 & 2.479089 & -0.028868 & 0.000000 \\ 1 & 1.264544 & -2.132520 & 0.000000 \\ 1 & -1.264544 & -2.132520 & 0.000000 \\ 1 & -2.479089 & -0.028868 & 0.000000 \\ 1 & -1.214544 & 2.161387 & 0.000000\end{array}$




$\begin{array}{lrrr}\Delta \mathrm{R}=0.15 \\ 6 & 0.659832 & 1.229464 & 0.000000 \\ 6 & 1.394663 & -0.043301 & 0.000000 \\ 6 & 0.734832 & -1.186163 & 0.000000 \\ 6 & -0.734832 & -1.186163 & 0.000000 \\ 6 & -1.394663 & -0.043301 & 0.000000 \\ 6 & -0.659832 & 1.229464 & 0.000000 \\ 1 & 1.202044 & 2.168604 & 0.000000 \\ 1 & 2.479089 & -0.043301 & 0.000000 \\ 1 & 1.277044 & -2.125303 & 0.000000 \\ 1 & -1.277044 & -2.125303 & 0.000000 \\ 1 & -2.479089 & -0.043301 & 0.000000 \\ 1 & -1.202044 & 2.168604 & 0.000000\end{array}$

$\Delta \mathrm{R}=0.2$

$\begin{array}{lrrr}6 & 0.647332 & 1.236681 & 0.000000 \\ 6 & 1.394663 & -0.057735 & 0.000000 \\ 6 & 0.747332 & -1.178946 & 0.000000 \\ 6 & -0.747332 & -1.178946 & 0.000000 \\ 6 & -1.394663 & -0.057735 & 0.000000 \\ 6 & -0.647332 & 1.236681 & 0.000000 \\ 1 & 1.189544 & 2.175821 & 0.000000 \\ 1 & 2.479089 & -0.057735 & 0.000000 \\ 1 & 1.289544 & -2.118086 & 0.000000 \\ 1 & -1.289544 & -2.118086 & 0.000000 \\ 1 & -2.479089 & -0.057735 & 0.000000 \\ 1 & -1.189544 & 2.175821 & 0.000000 \\ & & & \\ \Delta \mathrm{R}=0.25 & & \\ & & & \\ 6 & 0.634832 & 1.243898 & 0.000000 \\ 6 & 1.394663 & -0.072169 & 0.000000 \\ 6 & 0.759832 & -1.171729 & 0.000000 \\ 6 & -0.759832 & -1.171729 & 0.000000 \\ 6 & -1.394663 & -0.072169 & 0.000000 \\ 6 & -0.634832 & 1.243898 & 0.000000 \\ 1 & 1.177044 & 2.183038 & 0.000000 \\ 1 & 2.479089 & -0.072169 & 0.000000 \\ 1 & 1.302044 & -2.110869 & 0.000000 \\ 1 & -1.302044 & -2.110869 & 0.000000 \\ 1 & -2.479089 & -0.072169 & 0.000000 \\ 1 & -1.177044 & 2.183038 & 0.000000\end{array}$




\section{BLE}

\begin{tabular}{|c|c|c|c|}
\hline \multicolumn{4}{|c|}{$\Delta R=-0.15$} \\
\hline 6 & 0.000000 & 1.244663 & 0.000000 \\
\hline 0 & 1.077910 & 0.622332 & 0.000000 \\
\hline 6 & 1.077910 & -0.622332 & 0.000000 \\
\hline 6 & 0.000000 & -1.244663 & 0.000000 \\
\hline 6 & -1.077910 & -0.622332 & 0.000000 \\
\hline 6 & -1.077910 & 0.622332 & 0.000000 \\
\hline 1 & 0.000000 & 2.329089 & 0.000000 \\
\hline 1 & 2.017050 & 1.164544 & 0.000000 \\
\hline 1 & 2.017050 & -1.164544 & 0.000000 \\
\hline 1 & 0.000000 & -2.329089 & 0.000000 \\
\hline 1 & -2.017050 & -1.164544 & 0.000000 \\
\hline 1 & -2.017050 & 1.164544 & 0.000000 \\
\hline \multicolumn{4}{|c|}{$\Delta R=-0.1$} \\
\hline 6 & 0.000000 & 1.294663 & 0.000000 \\
\hline 6 & 1.121211 & 0.647332 & 0.000000 \\
\hline 6 & 1.121211 & -0.647332 & 0.000000 \\
\hline 6 & 0.000000 & -1.294663 & 0.000000 \\
\hline 6 & -1.121211 & -0.647332 & 0.000000 \\
\hline 6 & -1.121211 & 0.647332 & 0.000000 \\
\hline 1 & 0.000000 & 2.379089 & 0.000000 \\
\hline 1 & 2.060351 & 1.189544 & 0.000000 \\
\hline 1 & 2.060351 & -1.189544 & 0.000000 \\
\hline 1 & 0.000000 & -2.379089 & 0.000000 \\
\hline 1 & -2.060351 & -1.189544 & 0.000000 \\
\hline 1 & -2.060351 & 1.189544 & 0.000000 \\
\hline
\end{tabular}

$\begin{array}{rrrr}6 & 0.000000 & 1.344663 & 0.000000 \\ 6 & 1.164512 & 0.672332 & 0.000000 \\ 6 & 1.164512 & -0.672332 & 0.000000 \\ 6 & 0.000000 & -1.344663 & 0.000000 \\ 6 & -1.164512 & -0.672332 & 0.000000 \\ 6 & -1.164512 & 0.672332 & 0.000000 \\ 1 & 0.000000 & 2.429089 & 0.000000 \\ 1 & 2.103652 & 1.214544 & 0.000000 \\ 1 & 2.103652 & -1.214544 & 0.000000 \\ 1 & 0.000000 & -2.429089 & 0.000000 \\ 1 & -2.103652 & -1.214544 & 0.000000 \\ 1 & -2.103652 & 1.214544 & 0.000000\end{array}$


$\Delta \mathrm{R}=0.05$

$\begin{array}{rrrr}6 & 0.000000 & 1.444663 & 0.000000 \\ 6 & 1.251115 & 0.722332 & 0.000000 \\ 6 & 1.251115 & -0.722332 & 0.000000 \\ 6 & 0.000000 & -1.444663 & 0.000000 \\ 6 & -1.251115 & -0.722332 & 0.000000 \\ 6 & -1.251115 & 0.722332 & 0.000000 \\ 1 & 0.000000 & 2.529089 & 0.000000 \\ 1 & 2.190255 & 1.264544 & 0.000000 \\ 1 & 2.190255 & -1.264544 & 0.000000 \\ 1 & 0.000000 & -2.529089 & 0.000000 \\ 1 & -2.190255 & -1.264544 & 0.000000 \\ 1 & -2.190255 & 1.264544 & 0.000000\end{array}$

$\Delta \mathrm{R}=0.1$

$\begin{array}{rrrr}6 & 0.000000 & 1.494663 & 0.000000 \\ 6 & 1.294416 & 0.747332 & 0.000000 \\ 6 & 1.294416 & -0.747332 & 0.000000 \\ 6 & 0.000000 & -1.494663 & 0.000000 \\ 6 & -1.294416 & -0.747332 & 0.000000 \\ 6 & -1.294416 & 0.747332 & 0.000000 \\ 1 & 0.000000 & 2.579089 & 0.000000 \\ 1 & 2.233556 & 1.289544 & 0.000000 \\ 1 & 2.233556 & -1.289544 & 0.000000 \\ 1 & 0.000000 & -2.579089 & 0.000000 \\ 1 & -2.233556 & -1.289544 & 0.000000 \\ 1 & -2.233556 & 1.289544 & 0.000000\end{array}$

$\Delta \mathrm{R}=0.15$

$\begin{array}{rrrr}6 & 0.000000 & 1.544663 & 0.000000 \\ 6 & 1.337717 & 0.772332 & 0.000000 \\ 6 & 1.337717 & -0.772332 & 0.000000 \\ 6 & 0.000000 & -1.544663 & 0.000000 \\ 6 & -1.337717 & -0.772332 & 0.000000 \\ 6 & -1.337717 & 0.772332 & 0.000000 \\ 1 & 0.000000 & 2.629089 & 0.000000 \\ 1 & 2.276858 & 1.314544 & 0.000000 \\ 1 & 2.276858 & -1.314544 & 0.000000 \\ 1 & 0.000000 & -2.629089 & 0.000000 \\ 1 & -2.276858 & -1.314544 & 0.000000 \\ 1 & -2.276858 & 1.314544 & 0.000000\end{array}$




\section{CLAMPING}

$\begin{array}{lrrr}\alpha=5 & & & \\ 6 & 0.004577 & 1.395370 & 0.000000 \\ 6 & 1.206111 & 0.701622 & 0.000000 \\ 6 & 1.206111 & -0.701622 & 0.000000 \\ 6 & 0.004577 & -1.395370 & 0.000000 \\ 6 & -1.210663 & -0.693714 & 0.000000 \\ 6 & -1.210663 & 0.693714 & 0.000000 \\ 1 & -0.089959 & 2.475661 & 0.000000 \\ 1 & 2.188927 & 1.159918 & 0.000000 \\ 1 & 2.188927 & -1.159918 & 0.000000 \\ 1 & -0.089959 & -2.475661 & 0.000000 \\ 1 & -2.098994 & -1.315698 & 0.000000 \\ 1 & -2.098994 & 1.315698 & 0.000000 \\ \alpha=10 & & & \end{array}$

$\begin{array}{rrrr}6 & 0.009629 & 1.397529 & 0.000000 \\ 6 & 1.205449 & 0.707079 & 0.000000 \\ 6 & 1.205449 & -0.707079 & 0.000000 \\ 6 & 0.009629 & -1.397529 & 0.000000 \\ 6 & -1.215052 & -0.690415 & 0.000000 \\ 6 & -1.215052 & 0.690415 & 0.000000 \\ 1 & -0.178700 & 2.465434 & 0.000000 \\ 1 & 2.224423 & 1.077957 & 0.000000 \\ 1 & 2.224423 & -1.077957 & 0.000000 \\ 1 & -0.178700 & -2.465434 & 0.000000 \\ 1 & -2.045748 & -1.387412 & 0.000000 \\ 1 & -2.045748 & 1.387412 & 0.000000 \\ & & & \\ \alpha=15 & & & \\ & & & \\ 6 & 0.015766 & 1.401541 & 0.000000 \\ 6 & 1.205859 & 0.714396 & 0.000000 \\ 6 & 1.205859 & -0.714396 & 0.000000 \\ 6 & 0.015766 & -1.401541 & 0.000000 \\ 6 & -1.221614 & -0.687113 & 0.000000 \\ 6 & -1.221614 & 0.687113 & 0.000000 \\ 1 & -0.264849 & 2.448747 & 0.000000 \\ 1 & 2.253075 & 0.994998 & 0.000000 \\ 1 & 2.253075 & -0.994998 & 0.000000 \\ 1 & -0.264849 & -2.448747 & 0.000000 \\ 1 & -1.988237 & -1.453710 & 0.000000 \\ 1 & -1.988237 & 1.453710 & 0.000000\end{array}$




$\begin{array}{lrrr}\alpha=20 & & & \\ 6 & 0.023822 & 1.408200 & 0.000000 \\ 6 & 1.207564 & 0.724681 & 0.000000 \\ 6 & 1.207564 & -0.724681 & 0.000000 \\ 6 & 0.023822 & -1.408200 & 0.000000 \\ 6 & -1.231347 & -0.683446 & 0.000000 \\ 6 & -1.231347 & 0.683446 & 0.000000 \\ 1 & -0.346819 & 2.426379 & 0.000000 \\ 1 & 2.274643 & 0.912837 & 0.000000 \\ 1 & 2.274643 & -0.912837 & 0.000000 \\ 1 & -0.346819 & -2.426379 & 0.000000 \\ 1 & -1.927863 & -1.513436 & 0.000000 \\ 1 & -1.927863 & 1.513436 & 0.000000 \\ & & & \\ \alpha=25 & & & \\ & & & \\ 6 & 0.035075 & 1.418750 & 0.000000 \\ 6 & 1.211092 & 0.739706 & 0.000000 \\ 6 & 1.211092 & -0.739706 & 0.000000 \\ 6 & 0.035075 & -1.418750 & 0.000000 \\ 6 & -1.246136 & -0.678985 & 0.000000 \\ 6 & -1.246136 & 0.678985 & 0.000000 \\ 1 & -0.422296 & 2.399504 & 0.000000 \\ 1 & 2.289139 & 0.834025 & 0.000000 \\ 1 & 2.289139 & -0.834025 & 0.000000 \\ 1 & -0.422296 & -2.399504 & 0.000000 \\ 1 & -1.866875 & -1.565428 & 0.000000 \\ 1 & -1.866875 & 1.565428 & 0.000000\end{array}$

\section{BOAT-LIKE}

$\begin{array}{lrrr}\alpha=5 & & & \\ & & & \\ 6 & -0.000241 & 1.394553 & 0.020335 \\ 6 & 1.207780 & 0.697048 & 0.020335 \\ 6 & 1.206222 & -0.696422 & -0.040494 \\ 6 & 0.000241 & -1.394553 & 0.020335 \\ 6 & -1.207780 & -0.697048 & 0.020335 \\ 6 & -1.206222 & 0.696422 & -0.040494 \\ 1 & -0.000951 & 2.478853 & 0.034430 \\ 1 & 2.147186 & 1.238561 & 0.034571 \\ 1 & 2.145093 & -1.238454 & -0.069176 \\ 1 & 0.000951 & -2.478853 & 0.034430 \\ 1 & -2.147186 & -1.238561 & 0.034571 \\ 1 & -2.145093 & 1.238454 & -0.069176\end{array}$




$\begin{array}{lrrr}\alpha=10 & & & \\ 6 & -0.000961 & 1.394131 & 0.040725 \\ 6 & 1.207761 & 0.696226 & 0.040725 \\ 6 & 1.201464 & -0.693680 & -0.080840 \\ 6 & 0.000961 & -1.394131 & 0.040725 \\ 6 & -1.207761 & -0.696226 & 0.040725 \\ 6 & -1.201464 & 0.693680 & -0.080840 \\ 1 & -0.003820 & 2.478073 & 0.068947 \\ 1 & 2.147938 & 1.235677 & 0.069195 \\ 1 & 2.139474 & -1.235213 & -0.138753 \\ 1 & 0.003820 & -2.478073 & 0.068947 \\ 1 & -2.147938 & -1.235677 & 0.069195 \\ 1 & -2.139474 & 1.235213 & -0.138753 \\ & & & \\ \alpha=15 & & & \\ 6 & -0.002172 & 1.393469 & 0.061254 \\ 6 & 1.207772 & 0.694861 & 0.061254 \\ 6 & 1.193472 & -0.689068 & -0.120820 \\ 6 & 0.002172 & -1.393469 & 0.061254 \\ 6 & -1.207772 & -0.694861 & 0.061254 \\ 6 & -1.193472 & 0.689068 & -0.120820 \\ 1 & -0.008593 & 2.476826 & 0.103094 \\ 1 & 2.149236 & 1.230900 & 0.103398 \\ 1 & 2.130030 & -1.229759 & -0.208181 \\ 1 & 0.008593 & -2.476826 & 0.103094 \\ 1 & -2.149236 & -1.230900 & 0.103398 \\ 1 & -2.130030 & 1.229759 & -0.208181 \\ & & & \\ \alpha=20 & & & \\ 6 & -0.003900 & 1.392615 & 0.082010 \\ 1 & & & \\ 1 & & & \\ 1 & -2.151137 & -1.224307 & 0.136753 \\ 6 & 1.207876 & 0.692948 & 0.082010 \\ 6 & 0.182198 & -0.682559 & -0.160191 \\ 6 & -1.207876 & -0.692948 & 0.082010 \\ 6 & -1.182198 & 0.682559 & -0.160191 \\ 1 & -0.015236 & 2.475194 & 0.136427 \\ 1 & 2.151137 & 1.224307 & 0.136753 \\ 6 & & 1.222072 & -0.277010\end{array}$




$\begin{array}{lrrr}\alpha=25 & & & \\ 6 & -0.006177 & 1.391660 & 0.103066 \\ 6 & 1.208177 & 0.690498 & 0.103066 \\ 6 & 1.167565 & -0.674110 & -0.198662 \\ 6 & 0.006177 & -1.391660 & 0.103066 \\ 6 & -1.208177 & -0.690498 & 0.103066 \\ 6 & -1.167565 & 0.674110 & -0.198662 \\ 1 & -0.023722 & 2.473314 & 0.168593 \\ 1 & 2.153744 & 1.216004 & 0.168913 \\ 1 & 2.099501 & -1.212119 & -0.344977 \\ 1 & 0.023722 & -2.473314 & 0.168593 \\ 1 & -2.153744 & -1.216004 & 0.168913 \\ 1 & -2.099501 & 1.212119 & -0.344977\end{array}$

\section{CHAIR-LIKE}

$\begin{array}{lrrr}\alpha=5 & & & \\ 6 & 0.000000 & 1.393466 & -0.020234 \\ 6 & 1.208028 & 0.697213 & 0.020294 \\ 6 & 1.208028 & -0.697213 & -0.020294 \\ 6 & 0.000000 & -1.393466 & 0.020234 \\ 6 & -1.208028 & -0.697213 & -0.020294 \\ 6 & -1.208028 & 0.697213 & 0.020294 \\ 1 & 0.000000 & 2.477991 & -0.015990 \\ 1 & 2.146876 & 1.239998 & 0.015945 \\ 1 & 2.146876 & -1.239998 & -0.015945 \\ 1 & 0.000000 & -2.477991 & 0.015990 \\ 1 & -2.146876 & -1.239998 & -0.015945 \\ 1 & -2.146876 & 1.239998 & 0.015945 \\ & & & \\ \alpha=10 & & & \\ & & & \\ 6 & 0.000000 & 1.389745 & -0.040348 \\ 6 & 1.208747 & 0.696873 & 0.040604 \\ 6 & 1.208747 & -0.696873 & -0.040604 \\ 6 & 0.000000 & -1.389745 & 0.040348 \\ 6 & -1.208747 & -0.696873 & -0.040604 \\ 6 & -1.208747 & 0.696873 & 0.040604 \\ 1 & 0.000000 & 2.474587 & -0.032332 \\ 1 & 2.146672 & 1.241444 & 0.032108 \\ 1 & 2.146672 & -1.241444 & -0.032108 \\ 1 & 0.000000 & -2.474587 & 0.032332 \\ 1 & -2.146672 & -1.241444 & -0.032108 \\ 1 & -2.146672 & 1.241444 & 0.032108\end{array}$




$\begin{array}{lrrr}\alpha=15 & & & \\ 6 & 0.000000 & 1.383524 & -0.060206 \\ 6 & 1.209988 & 0.696323 & 0.060933 \\ 6 & 1.209988 & -0.696323 & -0.060933 \\ 6 & 0.000000 & -1.383524 & 0.060206 \\ 6 & -1.209988 & -0.696322 & -0.060933 \\ 6 & -1.209988 & 0.696322 & 0.060933 \\ 1 & 0.000000 & 2.468898 & -0.049080 \\ 1 & 2.146342 & 1.243896 & 0.048438 \\ 1 & 2.146342 & -1.243896 & -0.048438 \\ 1 & 0.000000 & -2.468898 & 0.049080 \\ 1 & -2.146342 & -1.243896 & -0.048438 \\ 1 & -2.146342 & 1.243896 & 0.048438 \\ & & & \\ \alpha=20 & & & \\ 6 & & & \\ 6 & 1.211781 & 0.695565 & 0.081291 \\ 6 & 1.211781 & -0.695565 & -0.081291 \\ 6 & 0.000000 & -1.374770 & 0.079676 \\ 6 & -1.211781 & -0.695565 & -0.081291 \\ 6 & -1.211781 & 0.695565 & 0.081291 \\ 1 & 0.000000 & 2.460893 & -0.066359 \\ 1 & 2.145881 & 1.247393 & 0.064953 \\ 1 & 2.145881 & -1.247393 & -0.064953 \\ 1 & 0.000000 & -2.460893 & 0.066359 \\ 1 & -2.145881 & -1.247394 & -0.064953 \\ 1 & -2.145881 & 1.247394 & 0.064953 \\ & & & \\ \alpha=25 & & & \\ 6 & 0.000000 & 1.363426 & -0.098606 \\ 6 & 1.214164 & 0.694607 & 0.101679 \\ 6 & 1.214164 & -0.694607 & -0.101679 \\ 6 & 0.000000 & -1.363426 & 0.098606 \\ 6 & -1.214164 & -0.694607 & -0.101679 \\ 6 & -1.214164 & 0.694607 & 0.101679 \\ 1 & 0.000000 & 2.450521 & -0.084271 \\ 1 & 2.145278 & 1.251992 & 0.081689 \\ 1 & 2.145278 & -1.251992 & -0.081689 \\ & 0.000000 & -2.450521 & 0.084271 \\ & -2.145278 & -1.251992 & -0.081689 \\ 6.145278 & 1.251992 & 0.081689\end{array}$




\section{PYRAMIDALIZATION}

$\begin{array}{lrrr}\alpha=5 & & & \\ 6 & -0.000045 & 1.394809 & -0.013488 \\ 6 & -1.207972 & 0.697350 & -0.013489 \\ 6 & -1.207972 & -0.697486 & -0.013531 \\ 6 & 0.000045 & -1.394809 & -0.013488 \\ 6 & 1.207972 & -0.697350 & -0.013489 \\ 6 & 1.207972 & 0.697486 & -0.013531 \\ 1 & -0.000112 & 2.475173 & 0.081031 \\ 1 & -2.143603 & 1.237517 & 0.081030 \\ 1 & -2.143571 & -1.237703 & 0.080989 \\ 1 & 0.000112 & -2.475173 & 0.081031 \\ 1 & 2.143603 & -1.237517 & 0.081030 \\ 1 & 2.143571 & 1.237703 & 0.080989 \\ & & & \\ \alpha=10 & & & \\ 6 & -1.208367 & 0.697670 & -0.026902 \\ 6 & -1.208367 & -0.697670 & -0.026902 \\ 6 & 0.000000 & -1.395402 & -0.026919 \\ 6 & 1.208367 & -0.697670 & -0.026902 \\ 6 & 1.208367 & 0.697670 & -0.026902 \\ 6 & 0.000000 & 1.395402 & -0.026919 \\ 1 & -2.133496 & 1.231724 & 0.161453 \\ 1 & -2.133496 & -1.231724 & 0.161453 \\ 1 & 0.000000 & -2.463608 & 0.161435 \\ 1 & 2.133496 & -1.231724 & 0.161453 \\ 1 & 2.133496 & 1.231724 & 0.161453 \\ 1 & 0.000000 & 2.463608 & 0.161435 \\ & & & \\ \alpha=15 & & & \\ 6 & -1.209103 & 0.698100 & -0.040128 \\ 6 & -1.209103 & -0.698100 & -0.040128 \\ 6 & 0.000000 & -1.396263 & -0.040097 \\ 6 & 1.209103 & -0.698100 & -0.040128 \\ 6 & 1.209103 & 0.698100 & -0.040128 \\ 6 & 0.000000 & 1.396263 & -0.040097 \\ 1 & -2.116788 & 1.222046 & 0.240696 \\ 1 & -2.116788 & -1.222047 & 0.240696 \\ 1 & 0.000000 & -2.444307 & 0.240726 \\ & 2.116788 & -1.222046 & 0.240696 \\ & 2.116788 & 1.222047 & 0.240696 \\ & 0.000000 & 2.444307 & 0.240726\end{array}$




$\begin{array}{lrrr}\alpha=20 & & & \\ 6 & 1.210156 & -0.698711 & -0.053047 \\ 6 & 1.210156 & 0.698711 & -0.053047 \\ 6 & 0.000000 & 1.397479 & -0.053014 \\ 6 & -1.210156 & 0.698711 & -0.053047 \\ 6 & -1.210156 & -0.698711 & -0.053047 \\ 6 & 0.000000 & -1.397479 & -0.053014 \\ 1 & 2.093563 & -1.208626 & 0.318206 \\ 1 & 2.093563 & 1.208625 & 0.318207 \\ 1 & 0.000000 & 2.417483 & 0.318237 \\ 1 & -2.093563 & 1.208626 & 0.318206 \\ 1 & -2.093563 & -1.208625 & 0.318207 \\ 1 & 0.000000 & -2.417483 & 0.318237 \\ & & & \\ \alpha=25 & & & \\ & & & \\ 6 & -0.065558 & -0.699513 & -1.211536 \\ 6 & -0.065559 & 0.699512 & -1.211536 \\ 6 & -0.065591 & 1.399080 & 0.000000 \\ 6 & -0.065559 & 0.699512 & 1.211536 \\ 6 & -0.065558 & -0.699513 & 1.211536 \\ 6 & -0.065591 & -1.399081 & 0.000000 \\ 1 & 0.393431 & -1.191558 & -2.064031 \\ 1 & 0.393430 & 1.191557 & -2.064031 \\ 1 & 0.393390 & 2.383369 & 0.000000 \\ 1 & 0.393430 & 1.191557 & 2.064031 \\ 1 & 0.393431 & -1.191558 & 2.064031 \\ 1 & 0.393389 & -2.383368 & 0.000000\end{array}$


In Figures S1-S6, we have included the normalized values of the aromaticity indexes given in paper for all the distortions studied. They have been normalized according to the following formula:

$$
I_{N}=\frac{I-I_{\text {benzene }}}{I_{\text {benzene }}},
$$

where $I_{N}$ stands for the normalized index, $I$ for the aromaticity index in question, and $I_{\text {benzene }}$ for the value of the aromaticity index for benzene molecule. Since FLU is already defined in terms of the difference with respect to benzene, and providing $F L U_{\text {benzene }}=0$, we have left the value of FLU unchanged. On the other hand, only the indexes that report reasonable trends have been depicted.

The values of the normalized indexes help to scale the indexes around 0 , the value given to benzene. This way, we can compare not only the trend of the different aromaticity indexes, but also the magnitude of the change with the distortion applied. In this sense, the BLA and the pyramidalization are the distortions which exhibit most significant change of aromaticity indexes $\left(\Delta I_{N} \sim 0.5\right)$, followed by the BLE and the chair distortion $\left(\Delta I_{N} \sim 0.3\right)$, and the distortions least affected are the clamping and the boatlike ones $\left(\Delta I_{N} \sim 0.1\right)$. In this reasoning the values of HOMA for clamping have been omitted because they give so large variation with the distortion, which we are tempted to believe such finding must be wrong (see also discussion in the text).

\section{BLA}

As compared with the rest of the distortions, BLA is the one that reports the largest changes when going from index to index. For BLA distortion all NICS values report the same magnitude of change, similar also to that of FLU and PDI. KSCI and SCI show the most significant change with the bond length alternation. Altogether we can conclude that BLA is equally affected by in-plane and out-of-plane magnetic indexes, and non-bonding multicenter interactions may be somehow important, as KSCI and SCI give slightly different values.

\section{BLE}

The change of the aromaticity indexes with the size of the ring is of similar magnitude for the hardness and NICS(1)zz, while PDI changes more smoothly with the size of the 
ring. Although $\mathrm{NICS}(0)$ and FLU give the same trend for this distortion, the magnitude of change is larger for the magnetic index of aromaticity. Neither electronic indexes, nor magnetic ones agree, and is thus hard to extract further conclusions for this distortion. As already mentioned in the text BLE is indeed the most difficult distortion for the aromaticity indexes.

\section{Clamping}

While NICS(1) and KSCI report a similar change, the other magnetic aromaticity measure, NICS(1)zz, on one hand, and another electronic index, SCI, on the other, exhibit a larger variation with the clamping deformation. It seems to indicate that inplane NICS components, as well as non-connected multicenter contributions have some role on the overall process.

\section{Boat-like}

In this case KSCI and SCI report the same magnitude of change with the distortion. In this sense, the non-connected multicenter contributions play an irrelevant role in this distortion. However, the different values of NICS(0) and NICS(1) seem to indicate the obvious different contribution from the in-plane and the out-of-plane components of the magnetic indexes.

\section{Chair-like}

NICS $(0)$ values are much more affected by the chair-like distortion than the rest of the indexes. Namely, the other magnetic indexes, NICS(1) and NICS(1) $)_{z z}$, report a small change $\left(\Delta I_{N} \sim 0.1\right)$, which indicates that in-plane ring current is seriously affected by the distortion, as one could anticipate. On other hand, KSCI and SCI give the same values, therefore multicenter interactions play only a marginal role.

\section{Pyramidalization}

The magnetic indexes are especially sensible to this distortion. Particularly, the out-ofplane component of the magnetic indexes (see NICS(1) and its zz-component) seems to 
be the most affected. On the other hand, the electronic indexes and the hardness are only slightly affected with the pyramidalization of the carbon atoms, in agreement with previous studies on the subject (see the text).

In general, neither the electronic indexes, nor the magnetic ones agree in any distortion analyzed. The particular nature of each distortion may even lead to different results among indexes of the same kind. This finding puts forward, once again, the need for different aromaticity indexes even of the same nature, to surely assess the aromaticity of a particular compound.

Figure S1. Normalized values of the aromaticity indexes analyzed for the BLA distortion.

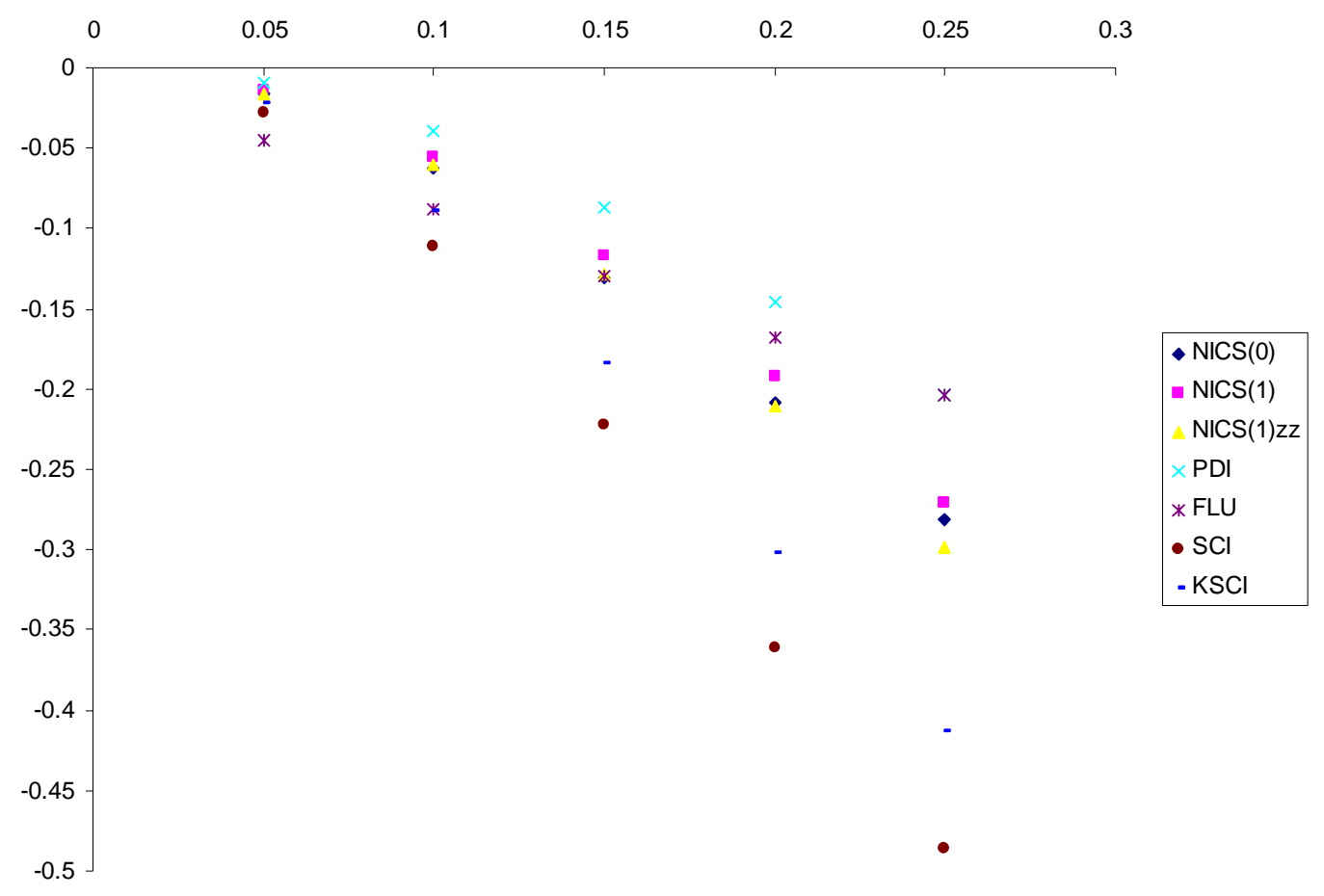


Figure S2. Normalized values of the aromaticity indexes analyzed for the BLE distortion.

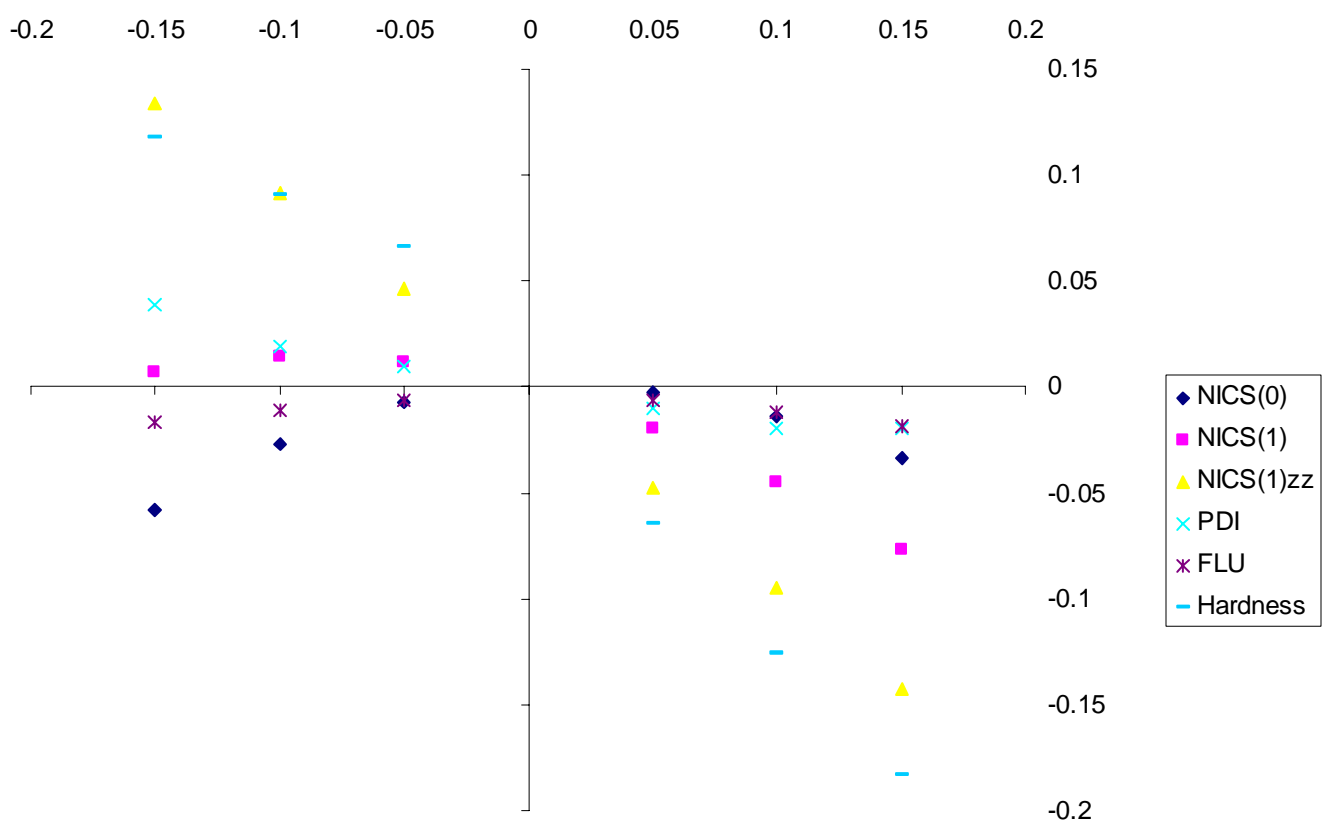

Figure S3. Normalized values of the aromaticity indexes analyzed for the clamping distortion.

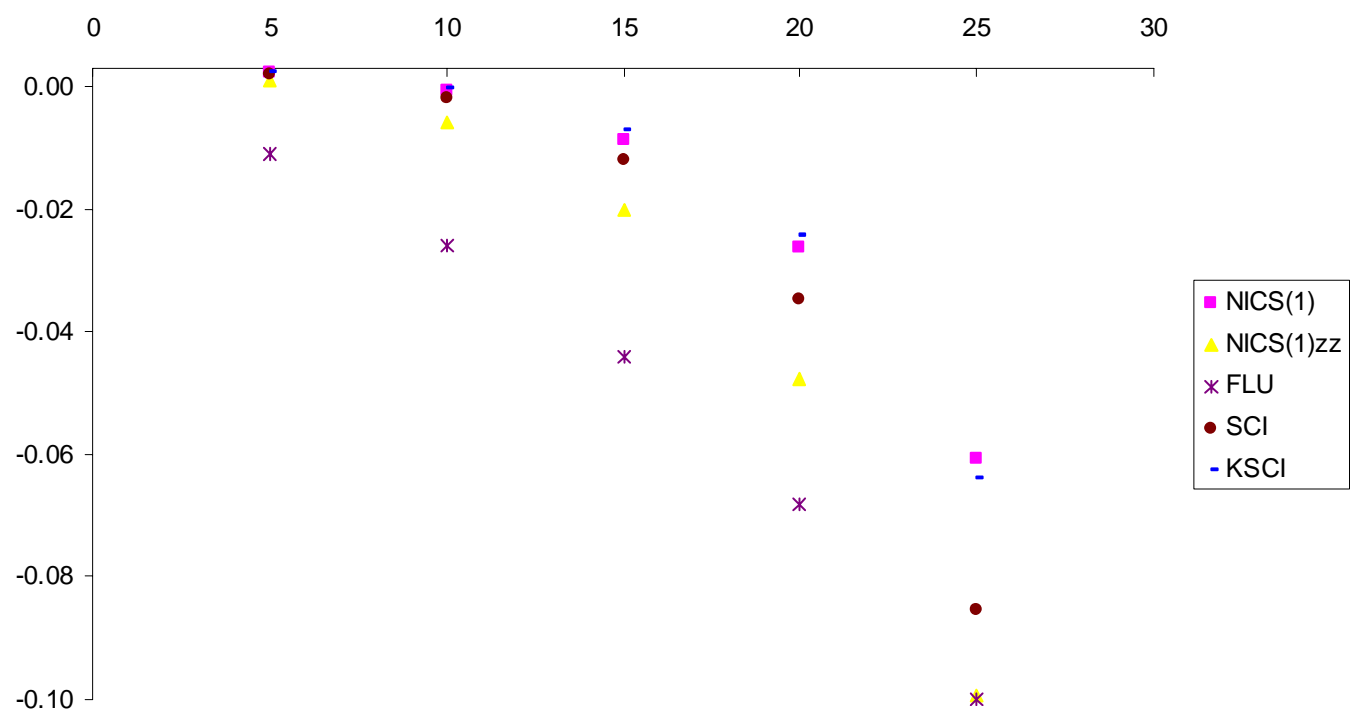


Figure S4. Normalized values of the aromaticity indexes analyzed for the boat-like distortion.

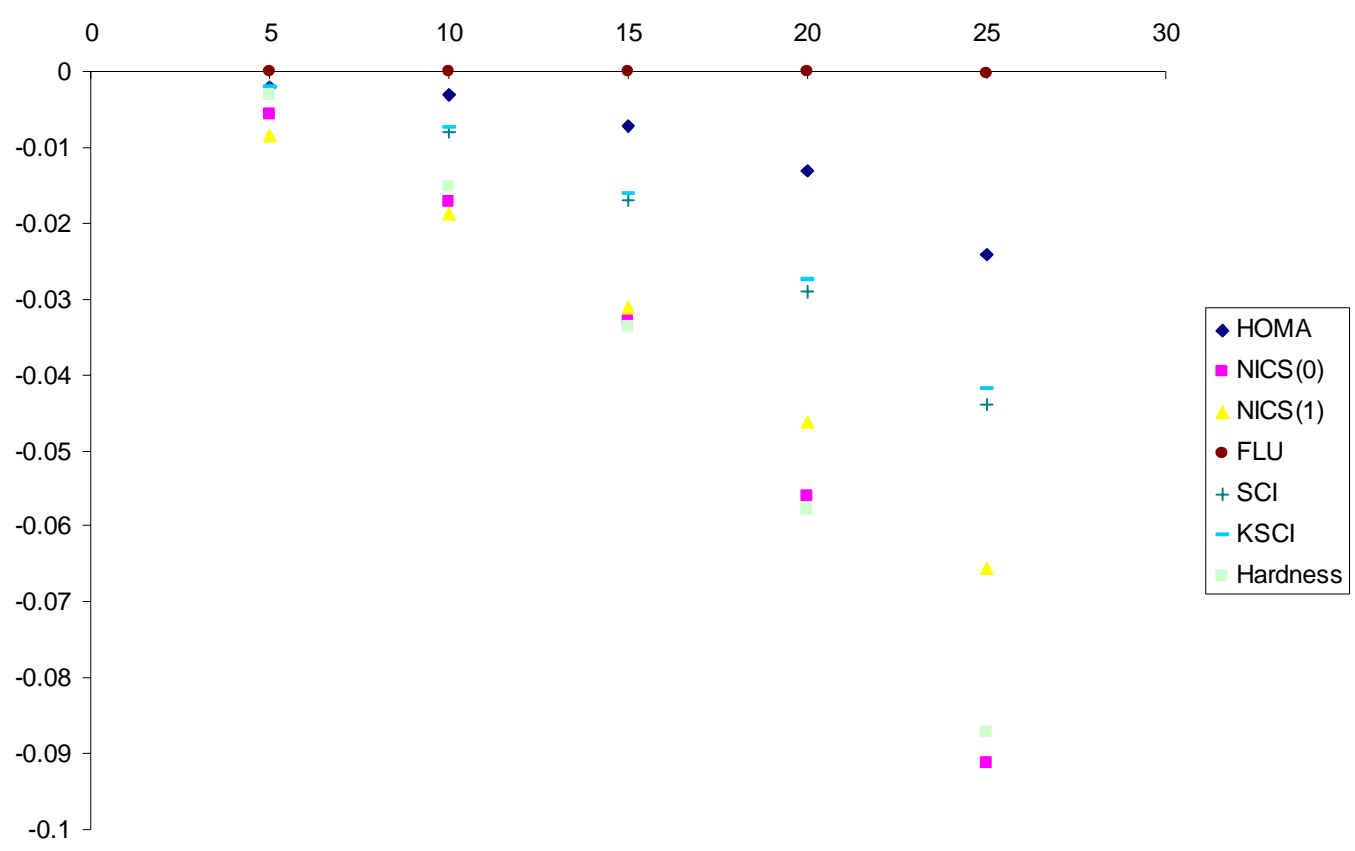

Figure S5. Normalized values of the aromaticity indexes analyzed for the chair-like distortion.

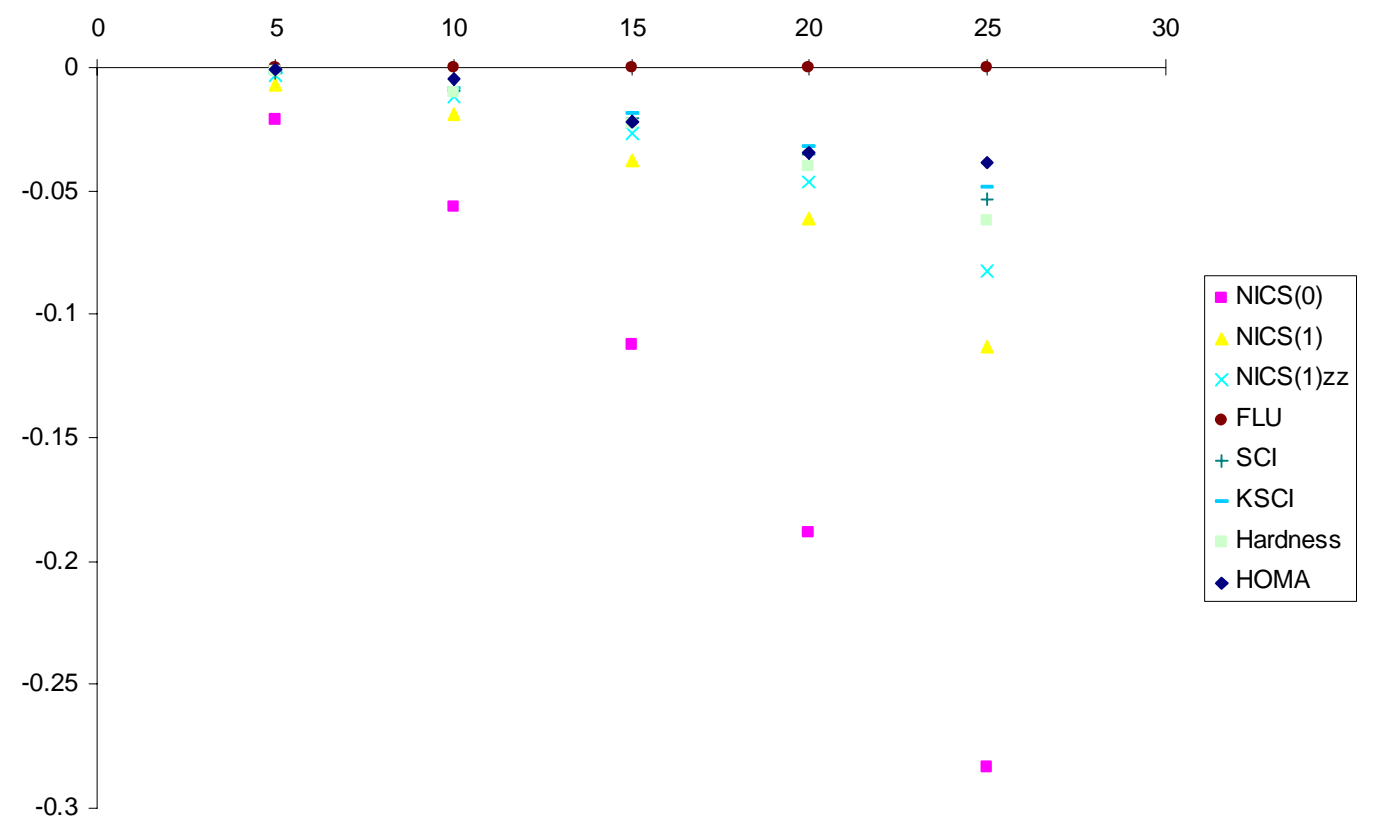


Figure S6. Normalized values of the aromaticity indexes analyzed for the pyramidalization distortion.

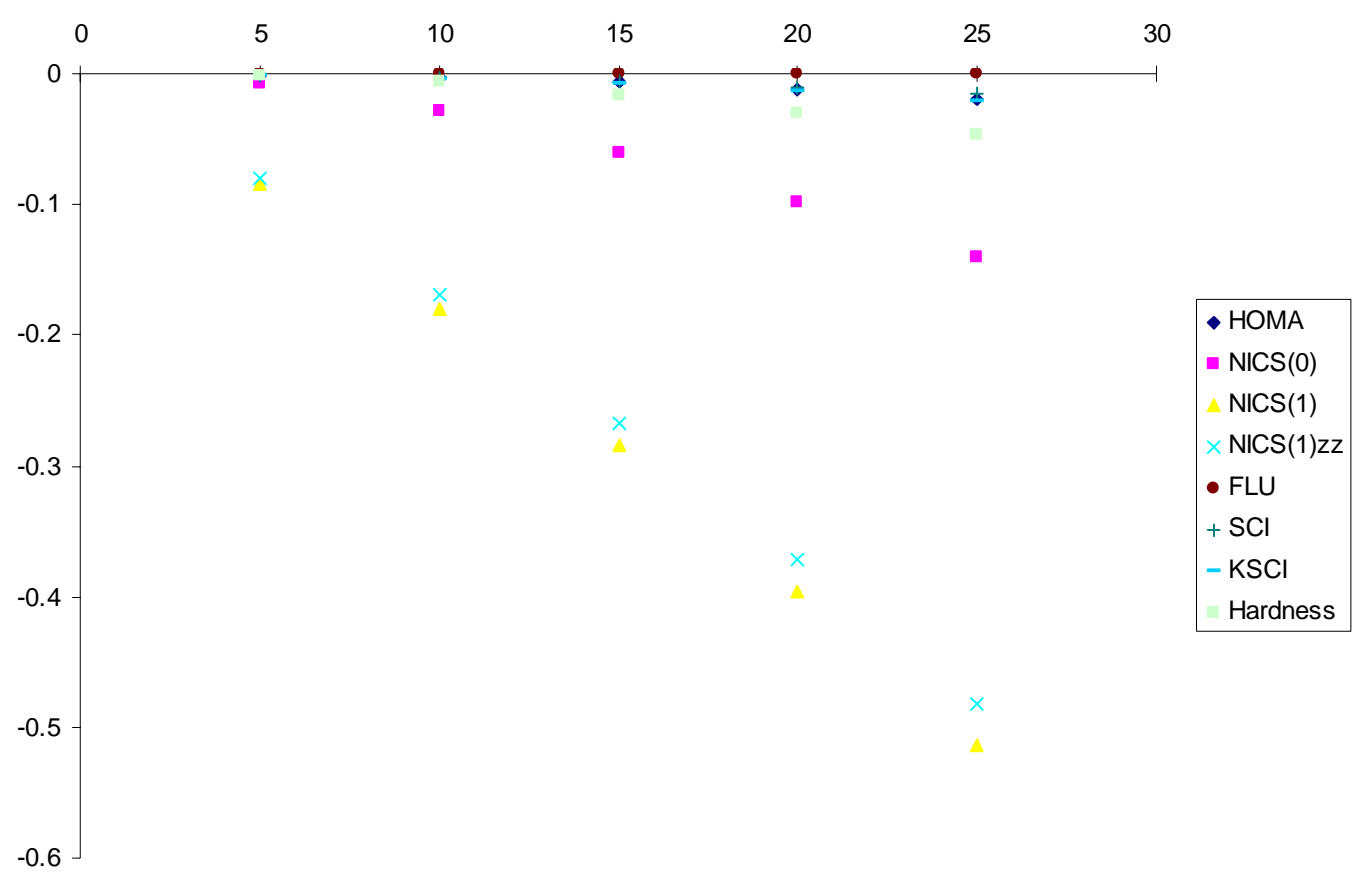

